\title{
The analysis of rheological damage of anchorage body based on visco-elasto-plastic model
}

\author{
Qing-duo Wang ${ }^{1}$, Feng-hai Yu ${ }^{1 *}$, Aleksei Renev ${ }^{2}$, Sergei Tsibaev ${ }^{2}$, and Xue-rui Yang \\ 1 State Key Laboratory of Mining Disaster Prevention and Control Co-founded by Shandong \\ Province and the Ministry of Science and Technology, Shandong University of Science and \\ Technology, Qingdao 266590, China \\ 2 T. F. Gorbachev Kuzbass State Technical University, 650000 Kemerovo, 28 Vesennyaya st., Russian \\ Federation
}

\begin{abstract}
In order to study the rheological damage of anchorage body, rheological damage model of anchorage body is established in this paper, and it is based on visco-elasto plastic model that is often used to simulate rock rheological characteristics. The expressions of creep constitutive equation and elastic modulus of anchorage body are obtained through the analysis of rheological damage model of anchorage body, and by the fitting calculation results, finding that the theoretical creep curve is matched with the experimental creep curve under certain conditions. The research conclusions have critical significance to the bolting support and design.
\end{abstract}

\section{Introduction}

The coal-measure strata formed an obvious layered distribution during the deposition and construction process. However, due to the influence of geological movement, the rock layer has a complicated structure and developed joints and fissures. The formation interface and joint fissures constitute the natural mechanical interface in the formation. After the roadway is excavated, the stress of the surrounding rock of the roadway will be redistributed. In this process, firstly, the separation layer will be gradually generated from these weak surfaces from bottom to top. Because of its own weight and horizontal stress, the lower rock layer produces rheology. At the same time of damage, the rock strata bends and sinks, so that the amount of separation is gradually expanded. If effective support measures are not taken in time, it is easy to cause the roof separation instability, which will cause severe consequences. So, it is valuable for the analysis of separation instability to study the rheological damage of the original rock and the anchored rock mass, and it also has certain guiding significance for on-site roadway bolt support. At present, there have been a lot of researches on the rheological damage of the original rock ${ }^{[1-4]}$, but relatively few researches on the rheological damage of anchored rock masses. Therefore, this paper establishes the anchor solid rheological damage model based on the Visco-elasto plastic model, which is commonly used

* Corresponding author: yufenghai2006@,163.com 
to simulate the rheological characteristics of rocks, and conducts analysis and experimental verification on it.

\section{The analysis of Rock initial damage}

After the roadway is excavated, the state of the rock mass will gradually change from the state of compression and shear to the state of tension and shear. Normally, the location of separation in a layered rock mass is where the bedding surface or joint cracks develop, which is generally caused by damage accumulation and expansion. The inhomogeneity of rock mass and the development of joints and fissures are determined by the mineral composition and structure inside the rock. The structure of the rock surface can be seen by scanning electron microscope, as shown in Fig 1.

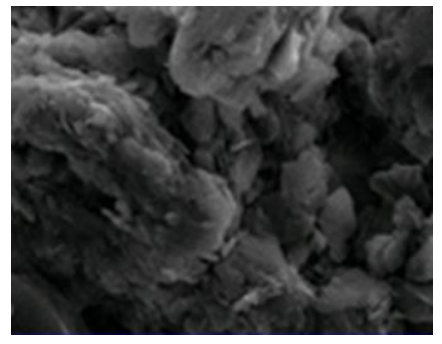

(a) Coarse sandstone slice image

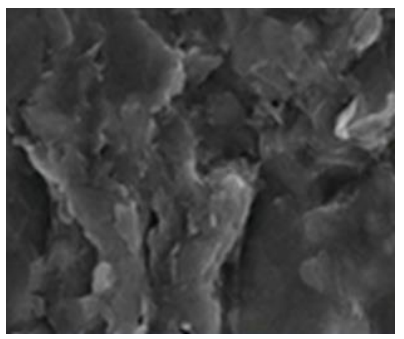

(b) Mudstone slice image

Fig. 1. Electron microscope scan results of different lithology rocks

It can be found from Fig 1 that different lithological rocks have different surface characteristics, and the same lithological rocks are not the same in different parts. Their grains, crystal size and shape, distribution status, and degree of mutual combination are all different, resulting the difference of the rock in quality. Due to these different meso-structure characteristics of the rock, it macroscopically appears as structural surfaces such as internal joints and cracks in the rock, which represents the initial damage state of the rock. It is inferred from this that the separation process of rock mass is the accumulation and expansion process of rock rheological damage.

\section{Rheological damage model of anchored body}

When using the element method to study the constitutive relationship of rocks, the commonly used models are Visco-elasto model, Burgers model, Bingham model, etc. According to the article ${ }^{[5]}$ about the analysis of comparing Burgers model with Visco-elasto model, it shows that Visco-elasto plastic model is more suitable for simulating the rheological characteristics of various rocks, while Burgers model is suitable for soft rocks. Therefore, this article selects the Visco-elasto plastic model for rheological damage analysis.

When analyzing the rheological damage process of rock strata supported by bolts, it is necessary to consider the role of bolt support. In order to simplify the treatment of the problem, it is assumed that the bolt support function is equivalent to a parallel high-stiffness spring ${ }^{[6]}$, and its equivalent stiffness is related to the bolt spacing and supporting strength and other bolt supporting parameters and support. If the effect is related, the anchor solid rheological damage model is composed of the Nishihara model and the Hooke elastomer model, referred to as the KBH model, as shown in Fig 2. 


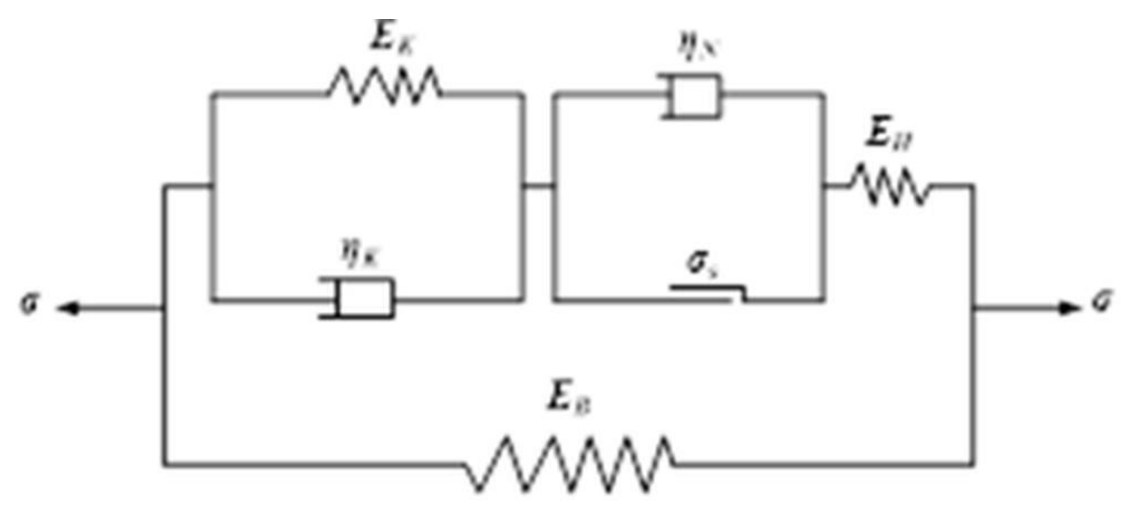

Fig. 2. Anchored General Nishihara Model

Assuming that the original rock model is represented by the subscript $\mathrm{R}$, the anchor rod is represented by $\mathrm{B}$, and the anchor solid is represented by $\mathrm{C}$. Then according to the principle of joint action of the anchor rod to strengthen the rock mass, the anchor rod in the anchor body does not cause instability failure, and the anchor rod and the original rock The deformations are coordinated, and the surrounding rock force is jointly carried by the anchor rod and the anchor solid composed of the original rock, then:

$$
\left.\begin{array}{l}
\varepsilon_{C}=\varepsilon_{R}=\varepsilon_{B} \\
\sigma_{C}=\sigma_{R}+\sigma_{B}
\end{array}\right\}
$$

The constitutive equation in the original rock model:

(1) When $\sigma_{R}<\sigma_{S}$

$$
\sigma_{R}+q_{0} \dot{\sigma}_{R}=q_{1} \varepsilon_{R}+q_{2} \dot{\varepsilon}_{R}
$$

Where, $q_{0}=\frac{\eta_{K}}{E_{H}+E_{K}}, \quad q_{1}=\frac{E_{H} E_{K}}{E_{H}+E_{K}}, \quad q_{2}=\frac{E_{H} \eta_{K}}{E_{H}+E_{K}}$.

(2) When $\sigma_{R} \geqq \sigma_{S}$

$$
q_{3}\left(\sigma_{R}-\sigma_{s}\right)+q_{4} \dot{\sigma}_{R}+\ddot{\sigma}_{R}=q_{5} \dot{\varepsilon}_{R}+\ddot{\varepsilon_{R}}
$$

The constitutive equation of the bolt model:

$$
\sigma_{B}=E_{B} \varepsilon_{B}
$$

Then for the anchor K-B-H model, when $\sigma_{R}<\sigma_{S}$, add formula (2) and (4):

$$
\sigma_{R}+q_{0} \dot{\sigma}_{R}+\sigma_{B}=q_{1} \varepsilon_{R}+q_{2} \dot{\varepsilon}_{R}+E_{B} \varepsilon_{B}
$$

When $\sigma_{R} \geqq \sigma_{s}$, add formular (3) and (4):

$$
q_{3}\left(\sigma_{R}-\sigma_{s}\right)+q_{4} \dot{\sigma}_{R}+\ddot{\sigma}_{R}+\sigma_{B}=q_{5} \dot{\varepsilon_{R}}+\ddot{\varepsilon}_{R}+E_{B} \varepsilon_{B}
$$

For the above-mentioned anchored generalized Visco-elasto plastic model (K-B-H model), the relationship shown in equation (1) as a whole is satisfied. Therefore, equations (1) can be substituted into equations (5) and (6). 
(1) When $\sigma_{c}<\left(E_{B}+E_{H}\right) \sigma_{s} / E_{H}$

$$
\sigma_{C}+q_{0} \dot{\sigma}_{C}=\left(q_{1}+E_{B}\right) \varepsilon_{C}+\left(q_{2}+q_{0} E_{B}\right) \dot{\varepsilon}_{C}
$$

(2) When $\sigma_{c} \geqq\left(E_{B}+E_{H}\right) \sigma_{s} / E_{H}$

$$
q_{3} \sigma_{C}+q_{4} \dot{\sigma}_{C}+\ddot{\sigma}_{C}=q_{3}\left(\sigma_{B}+\sigma_{s}\right)+\left(q_{5}+q_{4} E_{B}\right) \dot{\varepsilon}_{C}+\left(1+E_{B}\right) \ddot{\varepsilon}_{C}
$$

From the initial moment, $\mathrm{t}=0, \sigma \mathrm{c}=\sigma 0=$ const, the instantaneous strain of the model is $\varepsilon_{C}=\sigma_{0} /\left(E_{B}+E_{H}\right)$.

Therefore, the one-dimensional creep constitutive model of anchor is:

(1) When $\sigma_{0}<\left(E_{B}+E_{H}\right) \sigma_{s} / E_{H}$

$$
\varepsilon=\sigma_{0}\left[\frac{1}{q_{1}+E_{B}}+\left(\frac{1}{E_{H}+E_{B}}-\frac{1}{q_{1}+E_{B}}\right) \exp \left(-\frac{q_{1}+E_{B}}{q_{2}+q_{0} E_{B}} t\right)\right]
$$

(2) When $\sigma_{0} \geqq\left(E_{B}+E_{H}\right) \sigma_{s} / E_{H}$

When the initial value of stress $\sigma_{0}$ remains unchanged, that is, under the conditions of the creep experiment, with the increase of the anchor body strain $\varepsilon$, the bolt model stress $\sigma_{\mathrm{B}}$ gradually increases, resulting in the gradual decrease of the stress $\left(\sigma_{R}=\sigma_{0}-\sigma_{B}\right)$ on the Viscoelastoplastic model, Until the stress is reduced to $\sigma_{s}$, which makes the Bingham body partially fail. At this time, the Visco-elastoplastic model becomes the Burgers body equivalently, and the creep equation transforms into the form shown in equation (9). Therefore, according to formula (8), the creep constitutive equation in this case is:

$$
\varepsilon=\sigma_{0}\left[C(3)+\frac{\left(\sigma_{0}-\sigma_{B}-\sigma_{s}\right) q_{3}}{\left(q_{4} E_{B}+q_{5}\right) \sigma_{0}} t-\frac{C(4)\left(1+E_{B}\right)}{q_{4} E_{B}+q_{5}} \exp \left(-\frac{q_{4} E_{B}+q_{5}}{1+E_{B}} t\right)\right]
$$

In the formula, $\mathrm{C}(3)$ and $\mathrm{C}(4)$ are integral constants, which are determined by the initial conditions.

From the above analysis, it can be seen that the creep equation of anchor solids can be simplified as

$$
\varepsilon=\left(p_{0}+p_{1} t+p_{2} e^{p_{3} t}\right) \sigma_{c}
$$

In the formula, $p_{0} 、 p_{1}, p_{2} 、 p_{3}$ can be specifically determined according to specific actual conditions, especially $p_{l}$ exists under relatively high stress conditions, otherwise it is 0 .

According to the generalized Hooke's law, $E=\sigma / \varepsilon$. Under the initial stress $\sigma_{c}$, the elastic modulus expression of the anchor solid specimen at time $\mathrm{t}$ is

$$
E(t)=\frac{1}{p_{0}+p_{1} t+p_{2} e^{p_{3} t}}
$$

Therefore, the elastic modulus curve equation of the anchored body can be obtained according to the laboratory test, so as to be applied to the actual situation, and it provides a theoretical reference for the determination of the separation limit value of the roof of the boltsupported roadway.

\section{Test verification}

The method in the reference ${ }^{[7]}$ for the production of anchored specimens, during the creep test, the load application is divided into eight levels: $20 \%, 30 \%, 40 \%, 50 \%, 60 \%, 70 \%, 80 \%$, 
$85 \%$. The ultimate strength stress level and the failure mode of the anchored specimen are shown in Fig 4. Taking the $70 \% \sigma_{c}$ stress level in the creep curve of the anchored rock specimen as an example, the creep curve is fitted, and the theoretical value curve (according to formula (11)) and the test curve can be obtained by calculation, as shown in Fig 3.

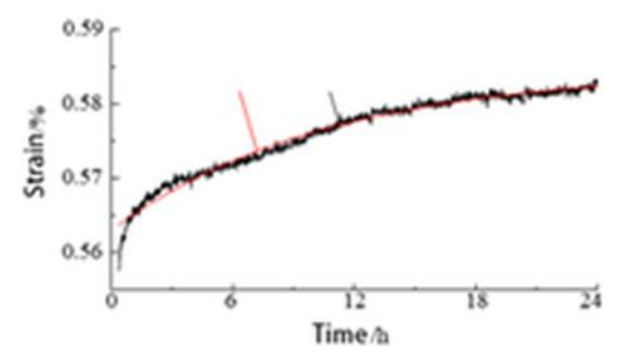

Fig. 3. Comparison chart of model theoretical value and experimental value

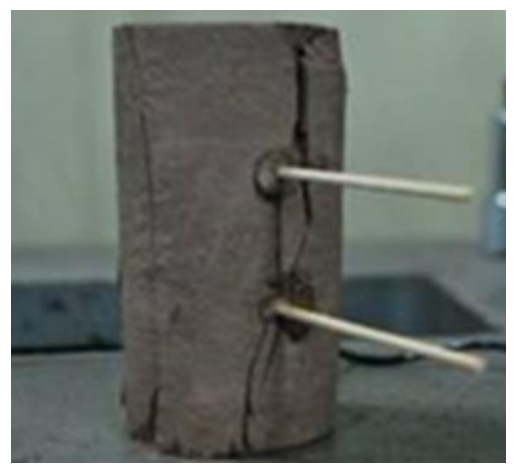

Fig. 4. Creep failure state of anchored specimen

It can be seen from Fig. 3 that the creep characteristics of the anchor body are analyzed by using the anchor body rheological damage model. It can be seen that the theoretical curve and the experimental curve are in good agreement, which verifies the rationality of the theoretical model. Therefore, under certain conditions, this model can be used for analysis and research.

\section{Conclusion}

The result of scanning electron microscopy shows that the interior of the rock is heterogeneous, which can be regarded as the initial damage of the rock. The failure process of the rock is caused by the continuous accumulation and expansion of damage, which can be reflected by the change of elastic modulus. By analyzing the rheological damage model of the anchored body, the creep constitutive equation of the anchored body is obtained: when $\sigma_{0}<\left(E_{B}+E_{H}\right) \sigma_{s} / E_{H}$, the creep constitutive equation is formula (9); When $\sigma_{0} \geqq\left(E_{B}+E_{H}\right) \sigma_{s} / E_{H}$, the creep constitutive equation is formula (10). The anchor creep equation can be simplified to formula (11), and the expression of the anchor's elastic modulus is formula (12). The theoretical creep curve (according to formula (11)) is obtained by calculation and fitting and the result obtained under certain conditions The test creep curves have good matching. Among them, the values of different lithological rock parameters $p_{0}, p_{1}, p_{2}$ and $p_{3}$ in the two formulas can be obtained by fitting the test data obtained from the corresponding rock creep test and applied in the field Appropriate corrections are needed. 


\section{Acknowledgments}

This work is supported by State Key Research Development Program of China (2017YFC0603001), National Natural Science Foundation of China(51704181).

\section{References:}

1. Wang De-sheng, Song Yong-jun, Chinese Journal of Rock Mechanics and Engineering, 26:6, 1081 (2007)

2. Yang Sheng-qi, Xu Peng, Chinese Journal of Rock Mechanics and Engineering, 36:10, $1846(2014)$

3. Wang Dong-sheng, Xu Chong-bang, etc, Chinese Journal of Rock Mechanics and Engineering, 27:8, 1594 (2008)

4. Song Yong-jun, Lei Sheng-you, Chinese Journal of Rock Mechanics and Engineering, 30:10, 1510 (2008)

5. Tao Bo, Wu Fa-quan, Chinese Journal of Rock Mechanics and Engineering. 24:17, 3165 (2005)

6. Zhao Tong-bin, Tan Yun-liang, etc. Analysis of rheological properties and control mechanism of anchored rock. Rock and soil mechanics, 33:6, 1730 (2012)

7. Zhao Tong-bin, Rock Creep Properties Creep Properties Properties Test In Deep Mine and Deformation Mechanism of Anchored Surrounding Rock (2009) 